ORIGINAL STUDY

\title{
Schneiderian membrane adult vasculogenesis evaluated by CD31 and CD34 expression and morphological arrangement
}

\section{Andreea-loana Derjac-Arama', Codrut Sarafoleanu ${ }^{2}$, Mugurel Constantin Rusu',3, Anca Vereanu ${ }^{4}$}

${ }^{1}$ Division of Anatomy, Faculty of Dental Medicine, "Carol Davila" University of Medicine and

Pharmacy, Bucharest, Romania

${ }^{2}$ Division of Otorhinolaryngology, Faculty of Dental Medicine, "Carol Davila” University of Medicine and Pharmacy, Bucharest, Romania

${ }^{3}$ MEDCENTER - Center of Excellence in Laboratory Medicine and Pathology, Bucharest, Romania ${ }^{4}$ Division of Oral Implantology, Faculty of Dental Medicine, "Carol Davila” University of Medicine and Pharmacy, Bucharest, Romania

\section{ABSTRACT}

BACKGROUND. Few studies approached the process of blood vessels formation in the Schneiderian membrane. We aimed at investigating by immunohistochemistry the processes that are responsible for forming new blood vessels in the human Schneiderian membrane.

MATERIAL AND METHODS. We applied CD31 and CD34 markers on bioptic samples gathered from eight adult patients negative for malignant pathologies. Filopodia-projecting endothelial tip cells (ETCs) were found and indicated processes of sprouting angiogenesis. Also, CD31-expressing monocyte-derived cells were found being involved in processes of vasculogenesis. These cells were projecting filopodia, thus being assessed as endothelial progenitor tip cells (EpTCs). Aggregates of CD31+ EpTCs were also analyzed. Further stages of lumen acquisition and large diameter vessels formation, specific for vasculogenesis, were evaluated.

RESULTS. It resulted that, specifically within the maxillary sinus mucosa, vascular remodelling is equally ensured by adult vasculogenesis and sprouting angiogenesis.

CONCLUSION. This is, to our knowledge, the first evidence of adult vasculogenesis in the maxillary sinus mucosa, supported by bona fide bone marrow-derived CD31+ cells. The guidance mechanism of EpTCs protrusions needs further investigations for finding similarities, or dissimilarities, with the endothelial tip cells prolongations.

KEYWORDS: maxillary sinus, endothelial tip cells, respiratory mucosa, paranasal sinuses

\section{INTRODUCTION}

The term "vasculogenesis" was first used to describe de novo blood vessel formation in the embryo, by Risau and co-workers in $1988^{1}$. During the process of vasculogenesis discrete events occur, such as lineage formation of angioblasts/endothelial progenitor cells (EPCs), migration of these, their aggregation into cord-like structures, lumen formation, network formation, vascular fusion (i.e. the generation of large vessels from primary networks) and vessel stabilization ${ }^{2}$. During the process of vasculogenesis, individual cell movement is important, at least during coalescence of cells into aggregates ${ }^{2}$. Cells engaged in vascular fusion show protrusive activity, which is related either to lamellipodia, or to filopodia ${ }^{2}$ which are also characteristic for endothelial tip cells involved in active processes of sprouting angiogenesis ${ }^{3-6}$.

The Schneiderian membrane consists of the maxillary sinus mucosa facing the maxillary sinus cavity and lying over a deeper layer of periosteum-like connective tissue; the sinus mucosa is build up by a pseudostratified columnar ciliated epithelium and a well vascularised lamina propria ${ }^{7}$. 
There are few available studies, mostly experimental, which indicate that angiogenesis is seemingly responsible for remodelling the microvascular bed of the Schneiderian membrane during regenerative pro$\operatorname{cesses}^{8,9}$. However, we could not identify in situ studies of human samples to objectivate the specific guidance of angiogenic sprouts by tip cells, nor did we find evidence of de novo blood vessels formation by vasculogenesis. We therefore aimed at investigating by immunohistochemistry the processes that are responsible for forming new blood vessels in the human Schneiderian membrane.

\section{MATERIAL AND METHODS}

\section{Tissue samples}

Bioptic samples of human adult Schneiderian membrane were obtained during surgery from eight adult patients who were operated for non-malignant pathologies. Informed consent for samples used for research purposes was obtained from all patients prior to surgery. The study was conducted according to the general principles from the Declaration of Helsinki, Cairo revision. Tissue samples were processed for immunohistochemistry.

\section{Antibodies}

Primary antibodies for CD31 (clone JC70A, Dako, Glostrup Denmark, 1:50) and CD34 (clone QBEnd 10, Dako, Glostrup Denmark, 1:50) were used.

\section{Immunohistochemistry}

Tissue samples were fixed for 24 hours in buffered formalin $(8 \%)$ and were processed with an automatic histoprocessor (Diapath, Martinengo, BG, Italy) with paraffin embedding. Sections were cut manually at 3 $\mu \mathrm{m}$ and mounted on SuperFrost ${ }^{\circledR}$ electrostatic slides for immunohistochemistry (Thermo Scientific, Menzel-Gläser, Braunschweig, Germany). Histological evaluations used 3- $\mu$ m-thick sections stained with hematoxylin and eosin.

Sections were deparaffinised, rehydrated and rinsed in PBS buffer solution at $\mathrm{pH}$ 7.4. Retrieval by incubation in specific buffer was completed as follows: (a) for CD34: EDTA, pH 9; (b) for CD31: $0.01 \mathrm{M}$ citrate retrieval solution, $\mathrm{pH}$ 6. The standard $\mathrm{ABC}$ technique used a DAB protocol. Appropriate blocking of endogenous peroxidase was completed before immune labelling (Peroxidazed 1, Biocare Medical, Concord, CA, USA). Sections incubated with non-immune serum served as negative controls. The immune labelled sections were counterstained with Hematoxylin. The microscopic slides were analysed and micrographs were acquired and scaled using a Zeiss working station which was described elsewhere ${ }^{10}$.

\section{RESULTS}

On slides stained with hematoxylin-eosin, the general histology of the Schneiderian membrane was accurately identified. The sinus maxillary sinus mucosa consisted of an epithelium of respiratory type and the lamina propria, embedding glands and microvessels.

CD34 was expressed in vascular endothelial cells. Endothelial tubes projecting filopodia (Figure 1), but not exclusively, were found. Moreover, small protrusions suggestive for a lamellipodial morphology were observed. This finding reasonably indicated that, within the maxillary sinus membrane, processes of sprouting angiogenesis occur and are guided by endothelial tip cells.

Numerous stromal cells expressing CD31 were also found (Figure $2 \mathrm{~A}-\mathrm{C}$ ). In a first instance, these were assumed, on histological ground, being histiocytes deriving from blood monocytes. Noteworthy, this consist-

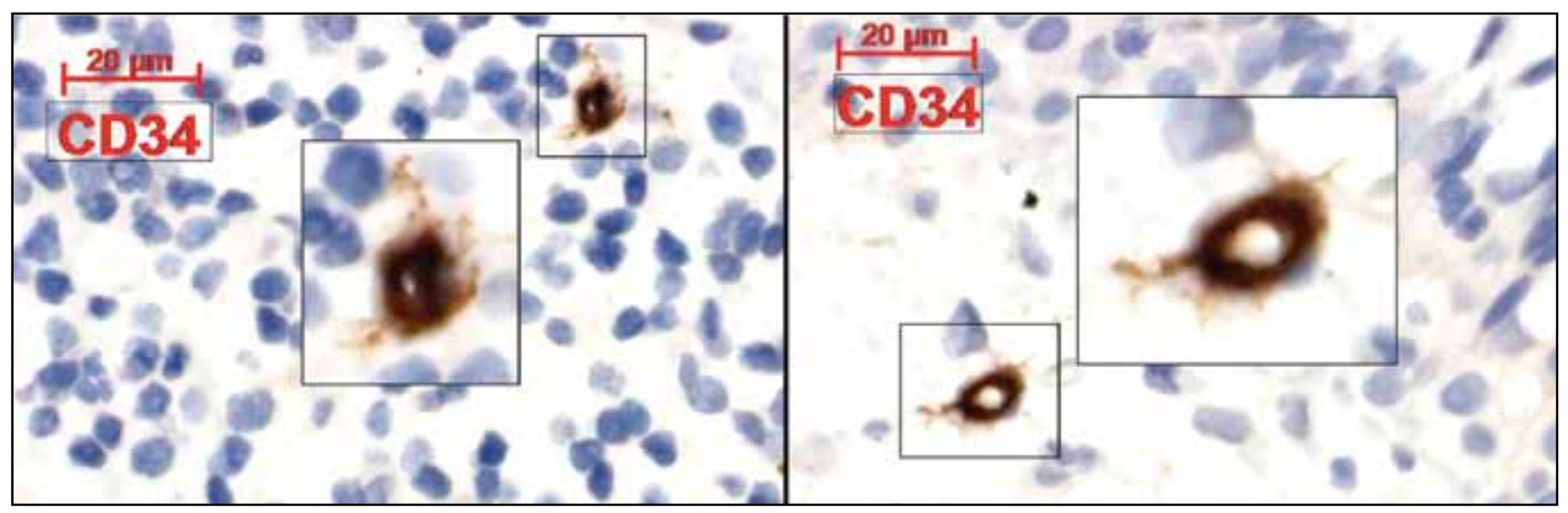

Figure 1 CD34 labelled adult Schneiderian membrane. Sprouting angiogenesis is identified by presence of filopodia-projecting (insets, magnified details) endothelial tip cells. 
ent population of CD31+ cells included mitotic and binucleated cells. Filopodia-projecting CD31+ cells, which were assumed being endothelial progenitor (tip) cells (EPtCs), were found; as in the case of endothelial tip cells, we could not firmly exclude these cells, so we also sent lamellipodial projections. Thus, CD31+ EPtCs showed tendency to aggregate and to form vascular lumina. Large vessels built up by CD31+/ CD34+ EPtCs were also found (Figure 2 B-D).

\section{DISCUSSIONS}

Several peculiar aspects determined us to consider that subsets of the CD31+ cells, the EPtCs, are effectively involved in processes of adult vasculogenesis in the Schneiderian membrane. These were the filopodia they were projecting in a similar manner to those of the ETCs, the tendency to aggregate of those cells and the large vessels they were forming, which were considered as resulted after vascular fusion of primary networks.

It was demonstrated that mesenchymal stem (stromal) cells (MSCs), as well as myeloid cells, enhance the de novo formation of blood vessels by endothelial colony-forming cells in various models of in vitro and in vivo vasculogenesis ${ }^{11}$. It is known that circulating monocytes are committed precursors for phagocytes, such as macrophages and dendritic cells; however, monocyte-derived multipotent cells were found able to differentiate in non-phagocytic cells, such as endothelium, bone, cartilage, fat, muscle and neuron ${ }^{12}$.

It resulted from our study that, specifically within the Schneiderian membrane (maxillary sinus mucosa), vascular remodelling is equally ensured by vasculogenesis and sprouting angiogenesis. This is, in our knowledge, the first evidence of adult vasculogenesis in the maxillary sinus mucosa, supported by bona fide bone marrow-derived CD31+ cells, these being previously indicated as highly angiogenic and vasculogenic ${ }^{13}$.

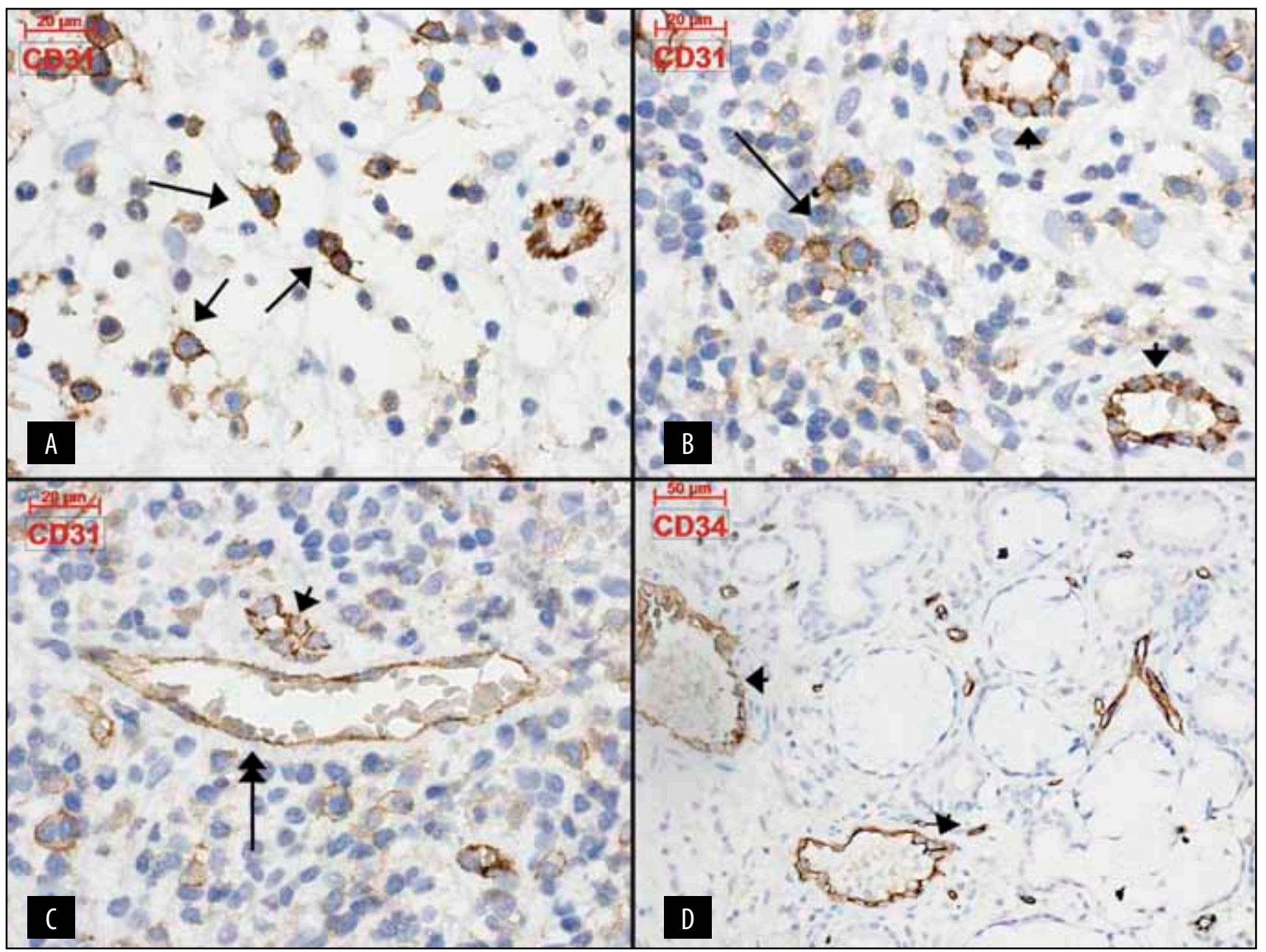

Figure 2 Adult Schneiderian membrane, labelled with CD31 (A-C) and CD34 (D) primary antibodies. There are identified stages of in situ vasculogenesis. In (A) CD31+ cells project filopodes (arrows) and show tendency to group. In (B) there are grouped CD31+ filopodia-projecting cells (arrow) as well as large vessels (arrowheads) which identify processes of vascular fusion. In (C) a newly formed vessel (arrowhead) neighbours a resident one (double-headed arrow). Large vessels, similar to those resulted from vascular fusion, are also CD34+ (D). 
Bone marrow-derived hematopoietic stem cells (HSCs) as well as their myeloid progeny could ensure vasculogenesis ${ }^{14}$. The more primitive HSCs express CD133 or CD34, while endothelial progenitor cells derived from monocytes express CD31 ${ }^{14}$. This indicates that adult vasculogenesis in the Schneiderian membrane is likely being supported by monocytes-derived endothelial cell progenitors.

Bone marrow-derived cells can home to sites of ischemia and ensure de novo formation of blood ves$\operatorname{sels}^{15}$. In this regard, the vascular regenerative potential of the Schneiderian membrane is double, extrinsic and intrinsic, supported, respectively, by vasculogenesis and angiogenesis. The term "endothelial progenitor tip cells" (EpTCs) could be used to distinguish between the endothelial tip cells involved in sprouting angiogenesis ${ }^{16-18}$ and filopodia-projecting monocytederived cells which aggregate to form new vessels. However, the guidance mechanism of EpTCs protrusions needs further investigations for finding similarities, or dissimilarities, with the endothelial tip cells prolongations.

\section{CONCLUSIONS}

In situ processes of de novo blood vessels formation maintain the vascular status of the Schneiderian membrane.

Acknowledgements: This paper is partly supported (author \#1) by the Sectorial Operational Programme Human Resources Development (SOPHRD), financed by the European Social Fund and by the Romanian Government under the contract number POSDRU/159/1.5/S/141531.

Conflicts of interests: None.

Contribution of authors: All authors have equally contributed to this work.

\section{REFERENCES}

1. Risau W., Sariola H., Zerwes H.G., et al. - Vasculogenesis and angiogenesis in embryonic-stem-cell-derived embryoid bodies. Development, 1988;102:471478.

2. Drake C.J., Fleming P.A., Argraves W.S. - The genetics of vasculogenesis. Novartis Found Symp, 2007;283:61-71; discussion -6, 238-41.

3. Phng L.K., Stanchi F., Gerhardt H. - Filopodia are dispensable for endothelial tip cell guidance. Development, 2013;140:4031-4040.

4. Rusu M.C., Poalelungi C.V., Vrapciu A.D., et al. - Endocardial tip cells in the human embryo - facts and hypotheses. PLoS One, 2015;10:e115853.

5. Rusu M.C., Didilescu A.C., Stanescu R., et al. - The mandibular ridge oral mucosa model of stromal influences on the endothelial tip cells: an immunohistochemical and TEM study. Anat Rec (Hoboken), 2013;296:350-363.

6. Stanescu R., Didilescu A.C., Jianu A.M., Rusu M.C. - Angiogenesis in the reparatory mucosa of the mandibular edentulous ridge is driven by endothelial tip cells. Rom J Morphol Embryol., 2012;53:375-8.

7. Srouji S., Kizhner T., Ben David D., Riminucci M., Bianco P., Livne E. - The Schneiderian membrane contains osteoprogenitor cells: in vivo and in vitro study. Calcif Tissue Int., 2009;84:138-145.

8. Forsgren K., Otori N., Stierna P., Kumlien J. - Microvasculature, blood flow, and vasoreactivity in rabbit sinus mucosa after surgery. Laryngoscope, 1999;109:562-568.

9. Zhang W., Wang X., Wang S., et al. - The use of injectable sonication-induced silk hydrogel for VEGF(165) and BMP-2 delivery for elevation of the maxillary sinus floor. Biomaterials, 2011;32:9415-9424.

10. Rusu M.C., Jianu A.M., Pop F., Hostiuc S., Leonardi R., Curca G.C. Immunolocalization of $200 \mathrm{kDa}$ neurofilaments in human cardiac endothelial cells. Acta Histochemica, 2012;114:842-845.

11. Watt S.M., Gullo F., van der Garde M., et al. - The angiogenic properties of mesenchymal stem/stromal cells and their therapeutic potential. Br Med Bull., 2013;108:25-53.

12. Seta N., Kuwana M. - Human circulating monocytes as multipotential progenitors. Keio J Med., 2007;56:41-47.

13. Kim H., Cho H.J., Kim S.W., et al. - CD31+ cells represent highly angiogenic and vasculogenic cells in bone marrow: novel role of nonendothelial CD31+ cells in neovascularization and their therapeutic effects on ischemic vascular disease. Circ Res., 2010;107:602-614.

14. Schatteman G.C., Awad O. - Hemangioblasts, angioblasts, and adult endothelial cell progenitors. Anat Rec A Discov Mol Cell Evol Biol., 2004;276:13-21.

15. Urbich C., Dimmeler S. - Endothelial progenitor cells: characterization and role in vascular biology. Circ Res., 2004;95:343-353.

16. Vrapciu A.D., Rusu M.C., Leonardi R., Corbu C.G. - Stem potentialities of the human iris - An in situ immunohistochemical study. Acta Histochem., 2014;116:1509-1513.

17. Rusu M.C., Motoc A.G., Pop F., Folescu R. - Sprouting angiogenesis in human midterm uterus and fallopian tube is guided by endothelial tip cells. Anat Sci Int., 2013;88:25-30.

18. Adams R.H., Eichmann A. - Axon guidance molecules in vascular patterning. Cold Spring Harb Perspect Biol., 2010;2:a001875. 